Revista peruana de biología 26(2): 259 - 264 (2019) doi: http://dx.doi.org/10.15381/rpb.v26i2.15730 ISSN-L 1561-0837; eISSN: 1727-9933

Universidad Nacional Mayor de San Marcos

\section{Ampliación del rango de distribución de la cotinga cariblanca Zaratornis stresemanni (Aves: Cotingidae) en el sur del Perú}

$\begin{array}{ll}\text { Presentado: } & 15 / 01 / 2019 \\ \text { Aceptado: } & 22 / 03 / 2019 \\ \text { Publicado online: } & 05 / 07 / 2019\end{array}$

Correspondencia:

*Autor para correspondencia

David Samata: david_esf@hotmail.com Daniel P. Cáceres Apaza: dcaceresapaza@gmail.com Gustavo Ordinola: gustav191@gmail.com Evaristo López: elopezt2001@yahoo.es

Otros datos de los autores / biografía: ORCID David Samata: 0000-0002-9587-2703 ORCID Daniel Cáceres Apaza: 0000-0002-2955-4061 ORCID Gustavo Ordinola: 0000-0001-5281-889X ORCID Evaristo López: 0000-0003-4269-3901

Citación:

Samata D., D.P. Cáceres Apaza, G. Ordinola, E. López. 2019. Ampliación del rango de distribución de la cotinga cariblanca Zaratornis stresemanni (Aves: Cotingidae) en el sur del Perú. Revista peruana de biología 26(2): 259 - 264 (Mayo 2019). doi: http:// dx.doi.org/10.15381/rpb.v26i2.15730

Palabras clave: Rango de distribución; Cotinga Cariblanca; Zaratornis stresemanni; Polylepis; Arequipa. Keywords: Distribution range; White-cheeked Cotinga; Zaratornis stresemanni; Polylepis; Arequipa.
Distribution range extension of the White-cheeked Cotinga Zaratornis stresemanni (Aves: Cotingidae) in southern Peru

\section{David Samata $^{1}$, Daniel P. Cáceres Apaza ${ }^{1 *}$, Gustavo Ordinola ${ }^{1}$, Evaristo López ${ }^{1,2}$}

1 Museo de Historia Natural (MUSA). Área de Ornitología. Universidad Nacional de San Agustín de Arequipa, Av. Alcides Carrión s/n, Arequipa, Perú.

2 Sección Zoología, Departamento Académico de Biología. Universidad Nacional de San Agustín de Arequipa, Av. Alcides Carrión s/n. Arequipa, Perú.

\section{Resumen}

La cotinga cariblanca Zaratornis stresemanni (Koepcke, 1954) (Aves: Cotingidae) es un ave endémica del Perú especialista de bosques de Polylepis. Actualmente se encuentra amenazada por la pérdida y degradación de su hábitat. En esta nota reportamos dos registros documentados al noroeste del departamento de Arequipa, en el sur de Perú, los cuales amplían en al menos 130 km su rango de distribución austral y corresponden a los primeros registros documentados de la especie para el departamento de Arequipa. Se sugiere que la cotinga cariblanca residiría en los bosques de Polylepis en el noroeste de Arequipa.

\section{Abstract}

The White-cheeked Cotinga Zaratornis stresemanni (Koepcke, 1954) (Birds: Cotingidae) is an endemic bird of Peru, specialist of Polylepis forests. Is currently threatened by habitat loss and degradation. In this note, we document records to the northwest of Arequipa department, in southern Peru. These records extend in at least $130 \mathrm{~km}$ the austral distribution range and correspond to the first documented records of the species for Arequipa department. We suggest that the White-cheeked Cotinga would be resident in the Polylepis forests in northwest Arequipa. 


\section{Introducción}

La familia Cotingidae (Passeriformes) es un grupo diverso de aves con amplia distribución en el Neotrópico, comprende 66 especies descritas (Snow 2004, Kirwan \& Green 2012). Las Cotingas se distribuyen principalmente en bosques de tierras bajas y bosques montanos, sin embargo algunas especies habitan en bosques de zonas áridas (Snow 2004, Kirwan \& Green 2012). Las Cotingas son principalmente frugívoras, aunque algunas especies pueden ser omnívoras o herbívoras (Snow 2004). La cotinga cariblanca Zaratornis stresemanni (Koepcke 1954) es un paseriforme frugívoro, no presenta dimorfismo sexual y está incluida en la subfamilia Phytotominae, donde se ubica como el linaje más ancestral (Tello et al. 2009, Berv \& Prum 2014). Es especialista de bosques de Polylepis (Fjeldsâ 2002), incluyendo bosques mixtos de Polylepis y Gynoxys, aunque ocasionalmente ocupa matorrales probablemente debido a la variación estacional en la disponibilidad de alimento (Parker 1981).

Zaratornis stresemanni se alimenta principalmente de frutos de plantas hemiparásitas de los géneros Tristerix y Ligaria (Parker 1981), sin embargo recientemente fue observada alimentándose de un arbusto de la especie Solanum nitidum (Barrio \& García-Olaechea 2015). Además, esta Cotinga depende de bosques de Polylepis parasitados por plantas del género Tristerix para realizar actividades reproductivas, tanto para la alimentación de los polluelos como para la construcción de nidos (Parker 1981). El sistema reproductivo social de la cotinga cariblanca es monógamo (Berv \& Prum 2014) y ambos adultos defienden un territorio durante la temporada reproductiva, donde probablemente el mes de marzo sea el auge de la época reproductiva (Parker 1981), adicionalmente, durante la época no reproductiva (entre agosto a setiembre) se observan grupos de alimentación de entre cuatro a diez individuos (Parker 1981).

Zaratornis stresemanni es una especie categorizada como vulnerable y se atribuye la amenaza a la degradación y pérdida de su hábitat (BirdLife International 2016). Se describe a esta especie como un ave pardusca, mayormente marrón, de apariencia fornida y de tamaño mediano como un zorzal (Turdus); característicamente presenta corona negra contrastante con prominente parche blanco en la cara; la garganta y parte superior del pecho es marrón-gris sin franjas; el resto del pecho y la espalda es mayormente de color ante con franjas oscuras prominentes; las plumas de las alas y cola son marrón oscuras con márgenes ocres (Schulenberg 2011).

Zaratornis stresemanni es endémica del Perú, se distribuye en la vertiente occidental de los Andes entre los 3800 a 4400 m de altitud (Schulenberg et al. 2010), entre los departamentos de La libertad, Ancash, Lima y Ayacucho (BirdLife International 2016), ocasionalmente desciende hasta los 2700 m (Parker 1981). La mayoría de los registros de la especie corresponden a la ladera oeste de los Andes en el centro del Perú (BirdLife International 2016, eBird 2018), mientras que los registros históricos en los extremos del rango de distribución fueron: por el norte, para la localidad de Yanac y la quebrada Turapac
(3650-4250 m de altitud), en el norte de la Cordillera Blanca, departamento de Ancash (Bond 1956, Parker 1981), y su registro más austral está en la Reserva Nacional de Pampa Galeras Bárbara D`Achille, departamento de Ayacucho (Parker 1981). Adicionalmente, Parker (1981) señaló un registro no confirmado para la localidad de Tayabamba, en el lado este del valle de Marañón, departamento de La Libertad, y propuso que eventualmente esta ave sería encontrada en bosques de Polylepis de la zona oeste del departamento, mientras que para el sur propuso que podría extenderse hasta el norte del departamento de Arequipa. Recientes registros confirmaron lo supuesto por Parker (1981) para el departamento de la Libertad, ampliando el rango de distribución norte de Z. stresemanni para ambos lados del valle del Marañón (eBird 2018). Sin embargo, hasta la actualidad no existen registros publicados que confirmen la presencia de la especie en el departamento de Arequipa. En esta nota reportamos dos registros confirmados de la Cotinga Cariblanca para el norte del departamento de Arequipa.

\section{Registros}

El primer registro corresponde a la localidad de Pirca $\left(15^{\circ} 26^{\prime} 01^{\prime \prime} \mathrm{S}\right.$ y $73^{\circ} 22^{\prime} 56^{\prime \prime} \mathrm{W}, 3775 \mathrm{~m}$ de altitud), distrito de Cahuacho, provincia de Caravelí, departamento de Arequipa. El 16 de junio del 2008, alrededor de las 9 horas un individuo de Cotinga Cariblanca fue capturado con una red de neblina, la red fue colocada dentro de un parche de Polylepis sp. cerca de una quebrada. El ambiente circundante se caracterizó por parches abiertos de Polylepis sp. y arbustos dispersos, además, se observó la presencia de plantas hemiparásitas (Loranthaceae) en algunos árboles de Polylepis sp., sin embargo no se determinó el género. El espécimen fue colectado y depositado en la colección científica del Museo de Historia Natural de la Universidad Nacional de San Agustín de Arequipa, MUSA 2206 (Fig. 1) como respaldo de su existencia en el departamento de Arequipa. Haciendo una inspección gonadal, al momento de la preservación, se determinó el sexo como macho, además, se determinó ausencia de muda, ausencia de límites de muda, coloración de plumaje típica de adulto, barras de desarrollo desalineadas y el $70 \%$ del cráneo neumatizado por lo que se infiere que se trataría de un adulto correspondiente al ciclo "DCB", según Johnson y Wolfe (2017), aunque no existen estudios detallados de los patrones de plumaje y muda para la especie.

El segundo registro fue cerca de la localidad de Saila $\left(15^{\circ} 18^{\prime} 55.44^{\prime \prime} \mathrm{S}\right.$ y $73^{\circ} 13^{\prime} 1.92^{\prime \prime} \mathrm{W}, 3784 \mathrm{~m}$ de altitud) dentro de la Reserva Paisajística Sub Cuenca del Cotahuasi, distrito de Tauría, provincia de La Unión, departamento de Arequipa. El 14 de setiembre del 2018 a las 8 horas con 34 minutos, se observó 2 individuos con prominente parche blanco en la mejilla y de corona negra contrastante (Fig. 2), características que corresponden a la Cotinga Cariblanca, estos individuos cruzaron la carretera que lleva al pueblo de Saila y se posaron sobre un arbusto de Solanum sp., uno de ellos estuvo realizando graznidos graves y ásperos. El hábitat donde fue registrado corresponde a un matorral con parches de pajonal, adicional- 

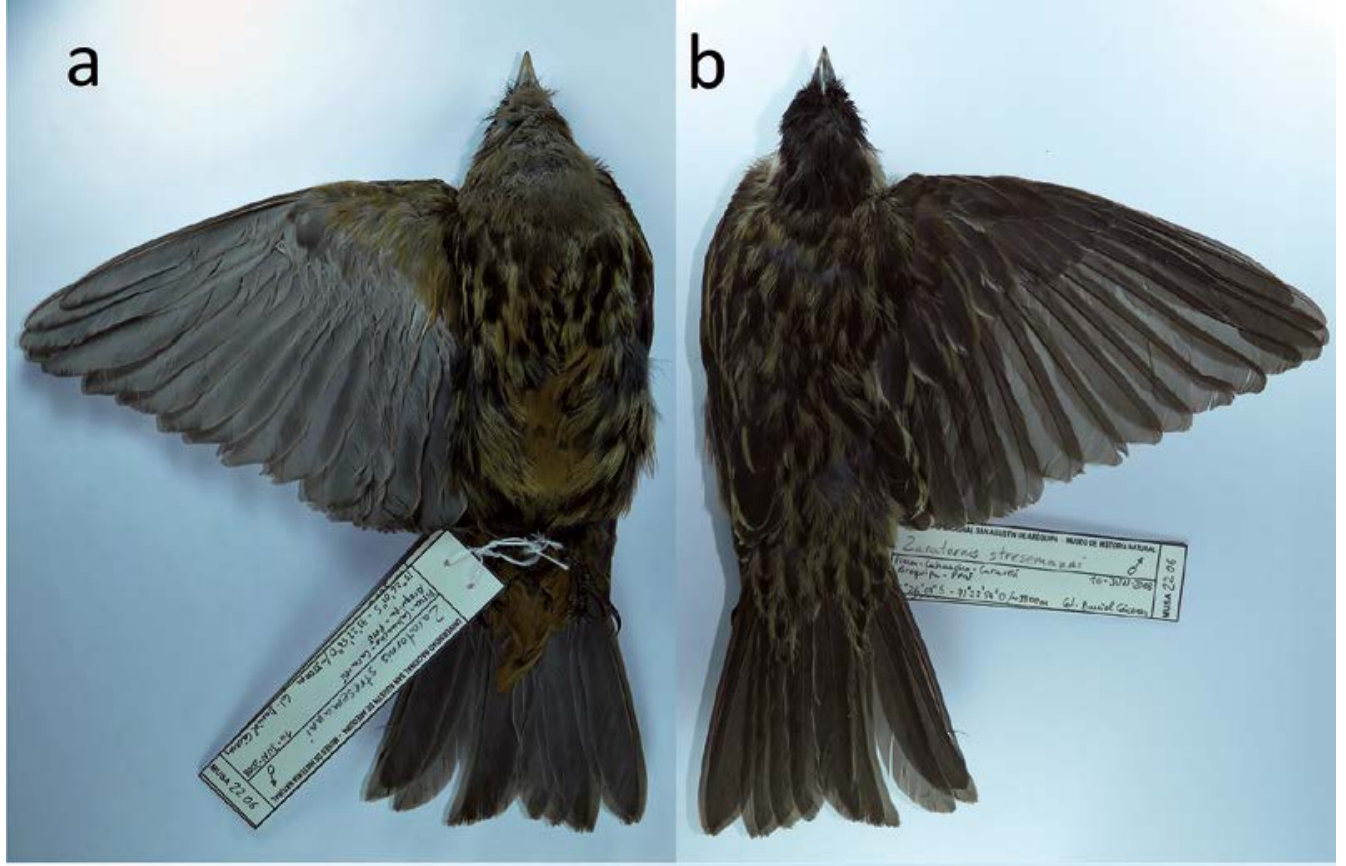

C

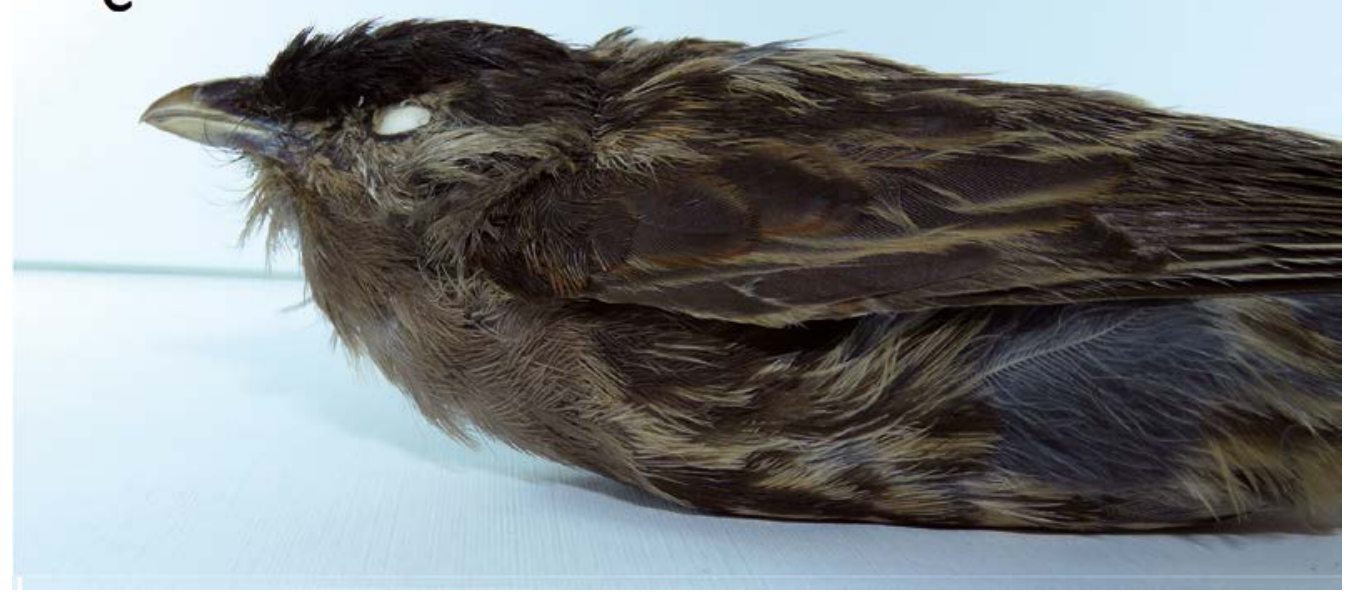

Figura 1. Individuo sub-adulto macho de Zaratornis stresemanni, colectado y depositado en el Museo de Historia Natural de la Universidad Nacional de San Agustín (MUSA-2206), se muestran las vistas ventral (a), dorsal (b) y lateral superior (c).

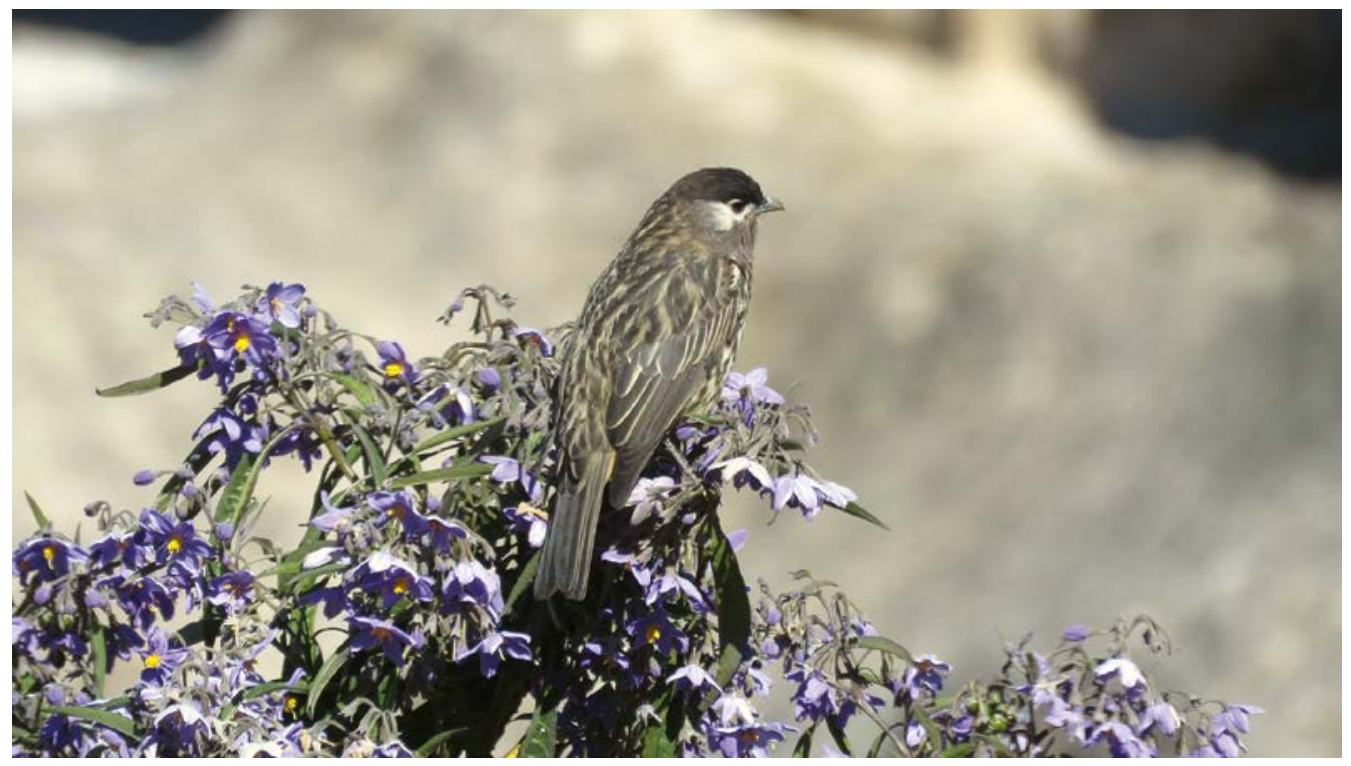

Figura 2. Individuo de Zaratornis stresemanni posado sobre arbusto de Solanum sp. 


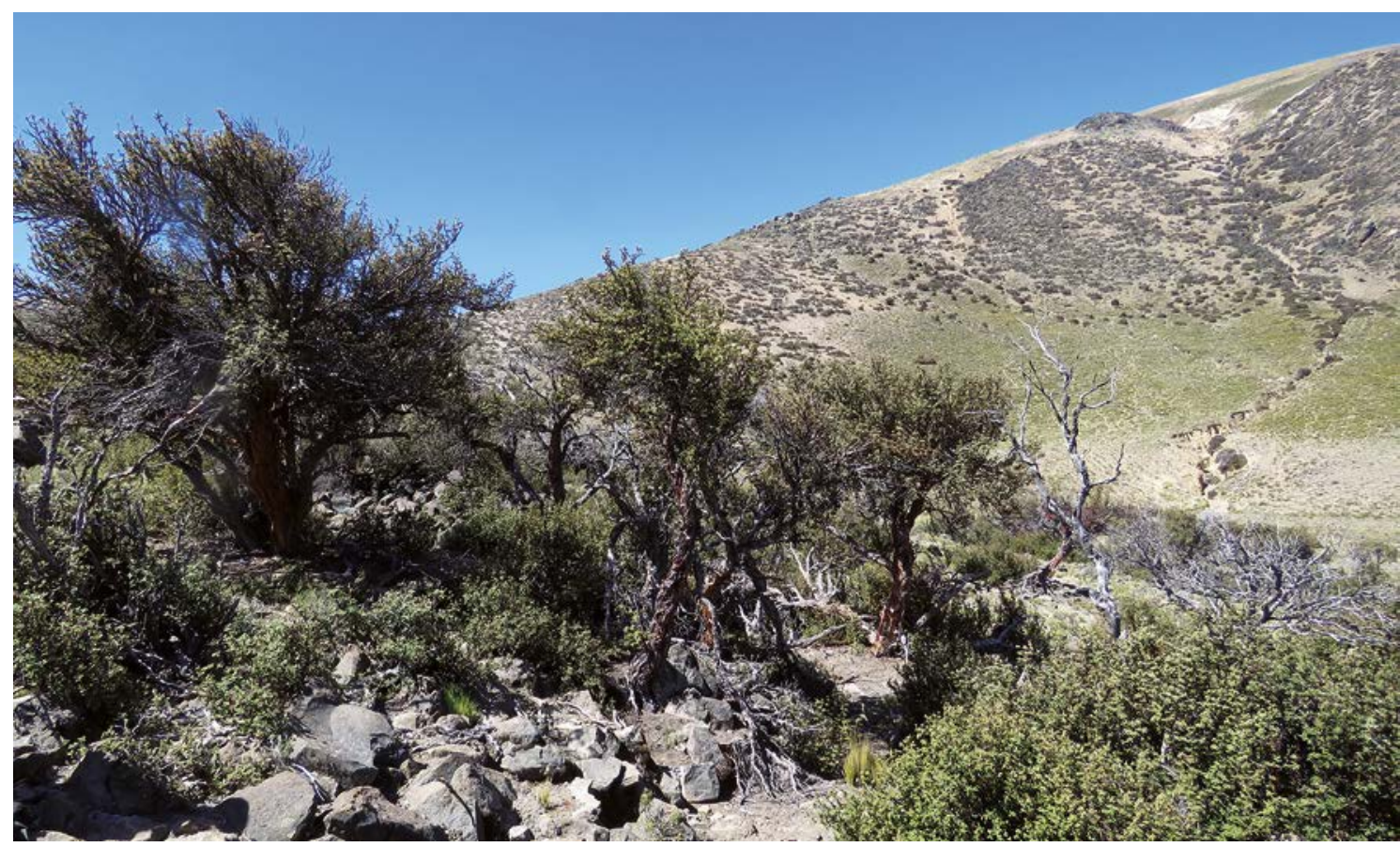

Figura 3. Parche de Polylepis sp. en el distrito de Tauría, Reserva Paisajística Sub Cuenca del Cotahuasi.

mente se registró la presencia de parches dispersos de Polylepis sp. a 8 kilómetros al suroeste del punto de observación, éstos estuvieron parasitados por plantas hemiparasitas (Loranthaceae) (Fig. 3).

Los registros reportados en esta nota amplían el rango de distribución austral de Z. stresemanni en más de 130 kilómetros al sur respecto de los registros publicados para el centro del departamento de Ayacucho (Parker 1981, eBird 2018) (Fig. 4). Ambos registros presentados aquí se ubican en la zona de vida correspondiente al matorral desértico Montano Subtropical (md-MS), cerca del límite con la zona de vida matorral desértico Montano Subalpino Subtropical (md-SaS), según la clasificación propuesta por ONERN (1976).

\section{Discusión}

Los registros reportados aquí son los más australes para Z. stresemanni y corresponden a los primeros registros documentados para el norte del departamento de Arequipa (Schulenberg et al. 2010). Estos registros amplían el rango de distribución de la especie en más de 130 km para el sur de la vertiente occidental de los Andes del Perú (Fig. 4). Se confirma lo supuesto por Parker (1981), quien propuso que la especie debería encontrarse en el extremo noroccidental del departamento de Arequipa en el límite con el departamento de Ayacucho.

Además, Parker (1981) menciona que Z. stresemanni habita en bosques de Polylepis y en bosques mixtos de Polylepis- Gynoxys desde los $3250 \mathrm{~m}$ hasta los $4300 \mathrm{~m}$ de altitud, pero durante la época seca (agosto hasta noviembre) también se podría encontrar a menor altitud hasta los 2700 m en bosques mixtos de Oreopanax, Escallonia y otras especies de árboles. Aunque nuestro segundo registro no corresponde con el hábitat preferido por la especie, este podría tratarse de un caso de dispersión en busca de recursos alimenticios. Además, cabe resaltar que existe un bosque de Polylepis sp. a 8 kilómetros al sur del sitio de avistamiento, lo cual podría señalar la residencia de esta especie en la zona. En consecuencia, la presencia de plantas hemiparásitas (Loranthaceae) en bosques de Polylepis, señalados para ambos registros, podría indicar que la especie se comporta como residente para el norte del departamento de Arequipa.

Después de la descripción de la cotinga cariblanca como especie (Koepcke 1954), son pocos los estudios que ampliaron el conocimiento de la historia natural para la especie (Parker 1981, Fjeldsâ 2002, Tello et al. 2009, Berv \& Prum 2014). Con el fin de comprender los factores que propician la presencia de la especie en el norte del departamento de Arequipa, estudios en la biología reproductiva, ecología trófica, requerimiento de hábitat y demografía serían necesarios para esclarecer su estatus de residencia e implementar acciones de conservación para esta especie en el sur del Perú.

\section{Literatura citada}

Barrio J. \& D. García-Olaechea. 2015. New records on the feeding niche for the White-cheeked Cotinga (Zaratornis stresemanni) in the high Andes. Boletín informativo UNOP, Vol 10. $\mathrm{N}^{\circ} 2-2015$

Berv J.S. \& R.O. Prum. 2014. A comprehensive multilocus phylogeny of the Neotropical cotingas (Cotingidae, Aves) with a comparative evolutionary analysis of breeding system and plumage dimorphism and a revised phylogenetic classification. Molecular Phylogenetics and Evolution, 81, 120-136. https://doi.org/10.1016/j. ympev.2014.09.001 


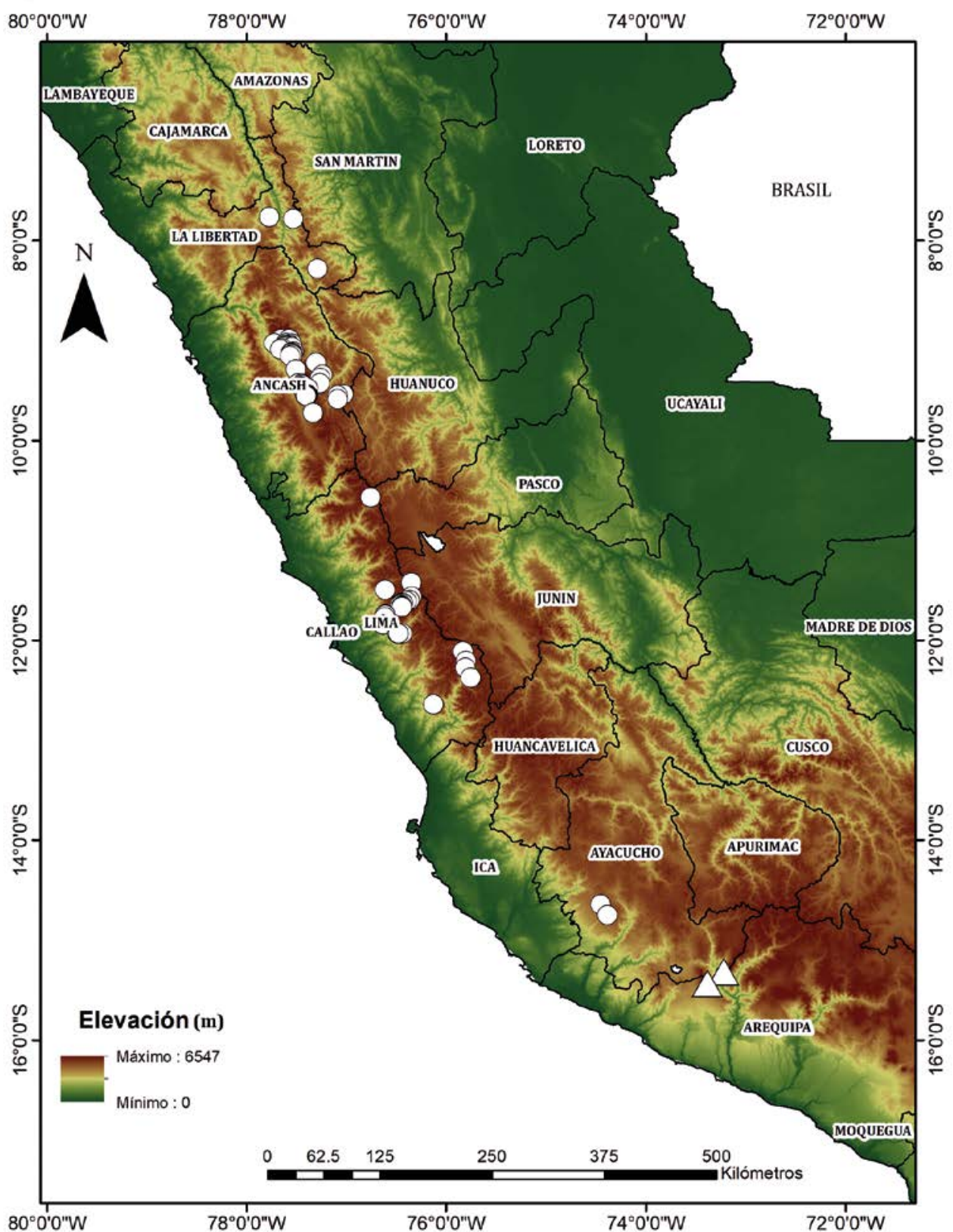

Figura 4. Distribución geográfica de Zaratornis stresemanni, los triángulos representan los registros reportados y los círculos los registros publicados en eBird (2018).

BirdLife International. 2016. (en línea). Zaratornis stresemanni. The IUCN Red List of Threatened Species 2016: e.T22700773A93795962. Acceso 01/10/2018.

Bond J. 1956. Additional notes on Peruvian birds II. Proceedings of the Academy of Natural Sciences of Philadelphia, 108, 227-279.

eBird. 2018. (en línea). eBird: An online database of bird distribution and abundance [web application]. eBird, Ithaca, New York. Acceso 01/10/2018.

De Juana E., J. Del Hoyo, M. Fernández-Cruz, et al. 2004. Nombres en castellano de las aves del mundo recomendados por la Sociedad Española de Ornitología (Novena parte: orden Passeriformes, familias Cotingidae a Motacillidae). Ardeola, 51(2), 491-499.

Fjeldsâ J. (2002). Key areas for conserving the avifauna of Polylepis forests. Ecotropica, 8, 125-131.
Johnson E.I. \& J.D. Wolfe. 2017. Molt in Neotropical birds: Life history and aging criteria. CRC Press.

Kirwan G.M. \& G. Green. 2012. Cotingas and Manakins. Princeton University Press, Princeton, NJ.

Koepcke M. 1954. Zaratornis stresemanni nov. gen. nov. spec., Un Cotingido nuevo del Perú. Publ. Mus. Hist. Natural "Javier Prado", Ser. A (Zoología), 16, 8 pp., 2 Abb.; Lima.

Schulenberg T.S. 2011. White-cheeked Cotinga (Zaratornis stresemanni), version 1.0. In Neotropical Birds Online (T. S. Schulenberg, Editor). Cornell Lab of Ornithology, Ithaca, NY, USA. https://doi.org/10.2173/ nb.whccot1.01; Acceso 01/10/2018.

ONERN. 1976. Mapa Ecológico del Perú. Guía explicativa. Lima: Oficina Nacional de Evaluación de Recursos Naturales. 
Pyle P. \& S.N. Howell. 1997. Identification guide to North American birds (No. C/598.297 P9).

Parker T.A. 1981. Distribution and biology of the White-cheeked Cotinga Zaratornis stresemanni, a high Andean frugivore. Bulletin of the British Ornithological Club, 101, 256-265.

Schulenberg T.S., D.F. Stotz, D.F. Lane, et al. 2010. Birds of Peru: revised and updated edition. Princeton University Press.

Snow D.W. 2004. Family cotingidae (Cotingidae). In: del Hoyo, J., Elliott, A., Christie, D.A. (Eds.). Handbook of the birds of the world. vol. 9. Cotingas to pipits and wagtails Lynx Edicions, Barcelona, Spain, pp. 32-108.

Tello J.G., R.G. Moyle, D.J. Marchese \& J. Cracraft. 2009. Phylogeny and phylogenetic classification of the tyrant flycatchers, cotingas, manakins, and their allies (Aves: Tyrannides). Cladistics, 25(5), 429-467. https://doi. org/10.1111/j.1096-0031.2009.00254.x

\section{Agradecimientos:}

A Margarita Esther Balvin Aguilar por elaborar el mapa de distribución, a Mauricio Ugarte Lewis por sus comentarios y consejos.

\section{Conflicto de intereses:}

Los autores no incurren en conflictos de intereses.

Rol de los autores:

DS, DCA, GO, realizaron aporte de datos, DS, DCA, GO y EL redactaron el trabajo y lo aprobaron.

Fuentes de financiamiento:

Los autores no manifiestan financiamiento.

Aspectos éticos / legales:

El trabajo no incluyo colectas ni manipulación de organismo. 